\title{
A influência da propaganda de medicamentos na automedicação
}

\author{
The influence of drug advertising on self-medication
}

Letícia da Silva Gimenes ${ }^{1}$, Sirlerne Carvalho Soares Garcia², Millena Pereira

Xavier $^{3}$, Natallia Moreira Lopes Leão ${ }^{4}$, Bruno Nunes do Vale ${ }^{5}$

RESUMO

Introdução: A propaganda de medicamentos é um conjunto de atividades que tem a intenção de persuadir através de informações sobre uma marca ou produto, visando aumentar a prescrição, aquisição e utilização do mesmo. Dessa forma termina por incentivar a automedicação que envolve a associação entre a escolha e uso de um medicamento, sem que haja acompanhamento médico, farmacêutico ou profissional de saúde habilitado acarretando casos graves de intoxicações. Objetivo: Verificar através de outros periódicos se a propaganda de medicamentos por meio dos meios de comunicação pode influenciar na pratica de automedicação. Métodos: Essa pesquisa foi através de periódicos publicados entre os anos de 2008 a 2016 nas bases de dados Scielo, Bireme e outros, sendo exclusas publicações com mais de dez anos. Desenvolvimento: Devido a grande audiência dos meios de comunicação, a indústria farmacêutica, enxergou a oportunidade de aumentar seus lucros através de propagandas de fármacos isentos de prescrição, de modo a aumentar consideravelmente o autoconsumo de medicamentos. É importante destacar a relevância da atenção farmacêutica, que tem por objetivo promover o uso racional de medicamentos, orientando e conscientizando sobre essa prática. O farmacêutico, com uma atuação ativa na dispensação, além de promover uma farmacoterapia eficiente, transforma a automedicação em prescrição farmacêutica, assumindo a responsabilidade de promotor de saúde favorecendo a população. Considerações Finais: Faz-se necessário que os órgãos de farmacovigilância atuem com campanhas de conscientização da população sobre a automedicação e seus riscos, e uma maior fiscalização sobre os anúncios que estão disponíveis.

Descritores: Propaganda de Medicamentos. Influência da Propaganda. Automedicação.

\section{ABSTRACT}

Introduction: Drug advertising is a set of activities that it intends to persuade through information about a brand or product, aiming to increase the prescription, acquisition and use of it. In this way, it ends up encouraging self-medication that involves the association between the choice and use of a medication, without medical, pharmaceutical or health care professional qualification leading to severe cases of intoxication. Objective: To verify through other journals whether the advertising of drugs through the media can influence the practice of self-medication. Methods: This research was published in journals published between the years 2008 and 2016 in thescielo, Biremeandothers databases, with publications with more than 10 years being excluded. Bibliographical review: Due to the large media audience, the pharmaceutical industry saw the opportunity to increase its profits through prescription drug advertisements, in order to greatly increase selfconsumption of drugs. It is important to highlight the relevance of pharmaceutical care, which aims to promote the rational use of medicines, guiding and raising awareness about this practice. The pharmacist, with an active role in the dispensation, besides promoting an efficient pharmacotherapy, transforms the self-medication into pharmaceutical prescription, assuming the responsibility of health promoter favoring the population. Final considerations: It is necessary for pharmacovigilance bodies to act with public awareness campaigns about self-medication and its risks, and a greater control over the ads that are available.

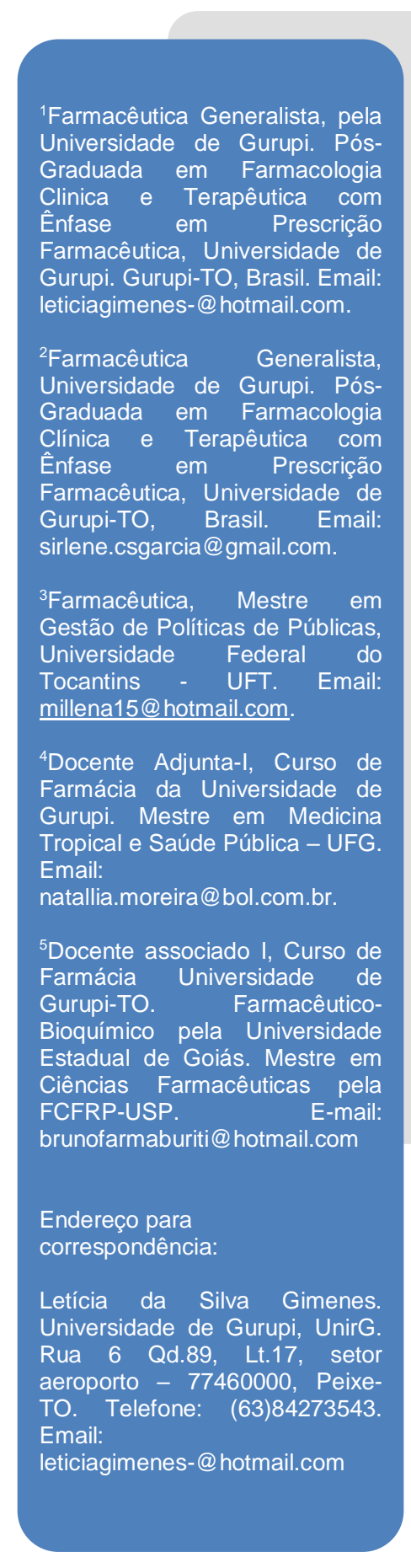

Descriptors: Medication Advertisement. Influence of Propaganda. Self-medication. 


\section{INTRODUCAOO}

A Resolução da Diretoria Colegiada no 96, de 17 de dezembro de 2008 (RDC 96/08) dispõe a respeito da propaganda de medicamentos, sendo esta determinada como um conjunto de atividades que tem a intenção de persuadir através de informações sobre uma marca ou produto, visando aumentar a prescrição, aquisição e utilização do mesmo'.

As propagandas de medicamentos segundo regras básicas, podem apresentar somente medicamentos isentos de prescrição (MIPs) ${ }^{1}$. Na maioria dos casos só é transmitido ao público os benefícios que o produto oferece, omitindo informações referente a sua segurança ${ }^{2}$. Desse modo a um aumento elevado no uso indiscriminado de medicamentos por automedicação e consequente intoxicações medicamentosas ${ }^{3,4}$.

A automedicação envolve a associação entre a escolha e uso de um medicamento, para tratar doenças e sintomas observados pelo indivíduo, sem que haja acompanhamento médico, farmacêutico ou profissional de saúde habilitado. Dentre as classes de medicamentos mais utilizados na automedicação estão os antitérmicos, antiinflamatório não hormonais, laxantes e antieméticos, sendo que o público que mais os utiliza de maneira indiscriminada são os idosos e adultos, principalmente quando esse tem um menor grau de escolaridade, e pouco conhecimento para buscar informações claras $^{5,6}$.

Este estudo teve como objetivo verificar através de outros periódicos se a propaganda de medicamentos, por meio dos meios de comunicação, pode influenciar na prática de automedicação.

\section{MATERIAIS E METODOS}

Essa pesquisa foi estruturada através de uma revisão da literatura do tipo exploratório de caráter bibliográfico, realizada entre os meses de janeiro a junho de 2017 , foram inclusos artigos entre os anos de 2008 e 2016.

Realizou-se coleta de dados em artigos publicados impressos e online, semelhante a temática central deste trabalho, contudo foram pesquisados artigos, disponíveis na íntegra, em revistas de saúde, nas bases de dados nacionais e internacionais tais como: Scielo, MedLine, Lilacs, PubMed, Periódico Capes, Sibnet USP, como em teses, dissertações, etc., as palavras chave utilizadas foram: automedicação, propaganda de medicamentos e mercado farmacêutico. 


\section{DESENVOLVIMENTO}

Devido à grande audiência dos meios de comunicação, a indústria farmacêutica, enxergou a oportunidade de aumentar seus lucros através de propagandas de fármacos isentos de prescrição ${ }^{7,8}$. Essas propagandas têm como objetivo influenciar o consumo de fármacos com informações convenientes, que facilitem as vendas, e muitas vezes são omitidos fatos importantes relacionados às reações adversas e contra indicação desse medicamento ${ }^{2,3,8}$.

A uma grande audiência das classes $C$ e $D$ nos programas de rádios que está presente em $90 \%$ dos lares brasileiros. Essa população muitas vezes com escolaridade incipiente não analisa de forma crítica as informações que são expostas se tornando alvo fácil para publicidade enganosa9. A propaganda ao divulgar os MIPs como produto de consumo tende a influenciar a automedicação agravando doenças, incentiva o uso de produtos ineficazes e termina por aumentar a quantidade de pacientes no sistema único de saúde por intoxicação 9 ,10,11.

De modo que foi necessária uma legislação específica para propagandas RDC 96 de 2008, através das normas exigidas para as publicidades, a regulamentação assegurasse a veracidade das informações nelas contidas por meio de alguns critérios obrigatórios como exemplo que conste de forma clara a contraindicação principal do fármaco, proibição da apresentação de propriedades curativas do medicamento quando no entanto é destinado para tratar sintomas e controlar doenças crônicas, e fica vetado utilizar expressões como "inócuo", "seguro" ou "produto natural"12,13.

A automedicação é uma iniciativa do indivíduo ou do seu responsável em fazer uso de um medicamento por sua conta e risco sem que haja acompanhamento de profissional responsável ${ }^{14}$.A escolha desses medicamentos normalmente ocorre por recomendação de pessoas leigas, por influência de prescrições anteriores e dificuldade de acesso aos serviços de saúde.Isso termina por aumentar o número de automedicações causando diversos casos de intoxicações farmacológicas ${ }^{3,15}$.

Através de uma pesquisa sobre automedicação por idosos Santos $^{6}$ verificou que dentre os fármacos mais utilizados por essa população esta os analgésicos e relaxantes musculares, entre os 461 casos constatados, 142 corresponde a estes medicamentos. É próprio do envelhecimento a diminuição de suas funções, alterando a biodisponibilidade do fármaco e dificultando a absorção, metabolização e eliminação levando a reações adversas $^{16}$. 
Agencia Nacional de Vigilância Sanitária (ANVISA) relata dentre as causas da automedicação a propaganda massiva de medicamentos, falta de conhecimento sobre efeitos adversos e ausência da orientação do farmacêutico. Os problemas decorrentes dessa prática é o uso inadequado por falta de diagnósticos e consequentes casos de intoxicações, alergia, interações resistência microbiana e disfarce de doenças mais graves $^{3}$.

A maioria dos casos de automedicação é principalmente com os medicamentos isentos de prescrição (MIPs) sendo que os mais utilizados estão os analgésicos e antitérmicos usados para tratar dor e febre. Uma pesquisa de campo realizada com 342 universitários de diversos cursos no Sul de Santa Catarina verificou que 96,5\% dos participantes praticavam o auto consumo de medicamentos sendo que 30\% eram da área de saúde ${ }^{14}$.Um resultado alarmante sabendo que os medicamentos e o principal causador de intoxicações farmacológicas ${ }^{17}$.

De acordo com o Sistema Nacional de Informações Toxicológicas (SINITOX), do Centro de Informação Científica e Tecnológica (CICT)/ Fundação Oswaldo Cruz(Fiocruz), foram notificados 3.969 (25\%) casos de intoxicações por medicamentos, nos anos de 2007 a 2009, estes encontram-se no topo da lista do SINITOX desde 1994, o resultado e preocupante quando $19 \%$ foram a óbito ${ }^{18}$.

Uma pesquisa realizada por Torres ${ }^{13}$, com uma amostra de 126 universitários na intenção de avaliar a influência da propaganda na automedicação indicou que 54,76\% dos participantes disseram sofrer influência da propaganda de (TV), 50,79\% confirmaram as vezes se automedicar e $60 \%$ concordam que a presença de um artista na propaganda aumenta a credibilidade do produto.

É importante destacar a relevância da atenção farmacêutica, que tem por objetivo promover o uso racional de medicamentos, orientando e conscientizando sobre essa prática, esse desfecho depende da presença do farmacêutico em período integral nas farmácias e drogarias ${ }^{19}$.

Um ganho muito válido contra a automedicação foi a implantação da resolução 586 de 29 de agosto de 2013 que regulariza a prescrição farmacêutica sobre medicamentos de venda livre ${ }^{20}$. Dessa forma o farmacêutico, com uma atuação ativa na dispensação, além de promover uma farmacoterapia eficiente, transforma a automedicação em prescrição farmacêutica, assumindo a responsabilidade de promotor de saúde favorecendo a população ${ }^{19}$. 


\section{CONSIDERACOES FINAIS}

A propaganda tem como finalidade evidenciar um produto e consequentemente aumentar suas vendas, e muitas vezes e deixado de lado informações sobre a segurança do medicamento evidenciando apenas seus benefícios e indicações.

Faz-se necessário que a Agência Nacional de Vigilância Sanitária (ANVISA) os que é o órgão de farmacovigilância brasileiro, atue com campanhas de conscientização da população sobre a automedicação e seus riscos, e uma maior fiscalização sobre os anúncios que estão disponíveis em farmácias, drogarias e até mesmo em redes sociais.

Com a nova atribuição para prescrição de MIPs, o farmacêutico poderá intervir na automedicação realizando a orientação medicamentosa do paciente em casos de doenças simples, evitando agravos e quando necessário encaminhando-o ao serviço médico.

\section{REFERENCIAS}

1 Brasil. Agencia Nacional de Vigilância Sanitária (ANVISA). Resolução da Diretoria Colegiada no 96 de dezembro de 2008. Expede as normas para publicação de propagandas de medicamentos. 2008 dez. 17.

2 Soares JCRS. Quando o anúncio é bom, todo mundo compra: o projeto Monitoração da propaganda de medicamentos no Brasil. Ciênc. Saúde Coletiva. 2008.13: 641- 649.

3 Alexandri AM, Foppa AA, Welter AC, Campos CMT, Cunha HP, Scherer MLS, et al. Propaganda de medicamentos: um desafio para todas as profissões. Rev. Bras. Farm. 2011.92(2): 66 - 70.

4 Trevisol DJ, Ferreira MBC, Karnopp ZMP. A propaganda de medicamentos em escola de medicina do Sul do Brasil. Ciênc. Saúde Coletiva. 2010.15 (Supl. 3): 3487 - 3496.

5 Oliveira AVC, Rocha FTR, Abreu SRO. Falência Hepática Aguda e Automedicação. Arq Bras Cir Dig. 2014.27(4): 294 - 297.

6 Santos TRA, Lima DM, Nakatani AYK, Pereira LV, Leal GS, Amaral RG. Consumo de medicamentos por idosos, Goiânia, Brasil. Rev. Saúde Pública. 2013.47(1): 94-103.

7 Rabello ET, Camargo Jr KR. Propagandas de medicamentos: a saúde como produto de consumo. Interface - Comunic. Saúde. Educ. 2012.41(16): 357-67.

8 Alves TNP, Mattos RA, Vieira RCPA. Medicamentos: conceitos, usos e problemas advindos do uso. Convibra Saúde. 2012: 1 - 18.

9 Batista AM, Carvalho MCRD. Avaliação da propaganda de medicamentos veiculada em emissoras de rádio. Ciênc. Saúde Coletiva 2013.18(2): 553 - 561.

10 Oenning D, Oliveira BV, Blatt CR. Conhecimento dos pacientes sobre os medicamentos prescritos após consulta médica e dispensação. Ciênc. Saúde Coletiva. 2011.(7): 3277 - 3283.

11 Nascimento ÁC. Propaganda de medicamentos para grande público: parâmetros conceituais de uma prática produtora de risco. Ciênc. Saúde Coletiva. 2010.15 (Supl. 3): 3423 - 3431. 
12 Brito MA. Leitura e discussão de reportagem sobre propaganda e uso off label de medicamentos com alunos de curso de Graduação em Farmácia. Rev. Bras. Farm. 2012. 93(3): 385 -391.

13 Torres LV. Influência da propaganda de medicamentos sobre o consumo em uma comunidade universitária de João Pessoa- PB. Monografia da biblioteca UFPB. 2016. 15-51.

14 Galato D, Madalena J, Pereira GB. Automedicação em estudantes universitários: a influência da área de formação. Ciênc. Saúde Coletiva. 2012 .17(12): 3323 - 3330.

15 Sousa HWO, Silva JL, Neto MS. A importância do profissional farmacêutico no combate à automedicação no Brasil. Revista Eletrônica de Farmácia. 2008. 5(1), 67-72.

16 Lyra Jr DP, Neves AS, Cerqueira KS, Marcellini PS, Marques TC, Barros JAC. Influência da propaganda na utilização de medicamentos em um grupo de idosos atendidos em uma unidade básica de saúde em Aracaju (SE, Brasil). Ciênc. Saúde Coletiva. 2010.15(Supl. 3): 3497 - 3505.

17 Silva RCG, Oliveira TM, Casimiro TS, Vieira KAM, Tardivo MT, Faria Jr M. et al. Automedicação em acadêmicos de medicina. Revistas USP. 2012.45(1): 5 - 11.

18 Brasil. Ministério da Saúde. Fundação Oswaldo Cruz. Sistema Nacional de Informações TóxicoFarmacológicas - SINITOX. Centro de Informação Científica e Tecnológicas. Casos, Óbitos e Letalidade de Intoxicação Humana por Região e Centro. 2007 - 2009.

19 Fernandes WS,Cembranelli JC. Automedicação e o uso irracional de medicamentos: o papel do profissional farmacêutico no combate a essas práticas. Revista Univap. 2015.21(37): 2237 - 1753.

20 BRASIL. CONSELHO FEDERAL DE FARMÁCIA. Resolução ㄲo. 586 de 29 de agosto DE 2013. Regulamenta a prescrição farmacêutica e dá outras providências. 1 - 12 . 